\title{
О ТЕХНОЛОГИИ ВЫЯВЛЕНИЯ УЧАСТКОВ ТРУБОПРОВОДОВ С ИЗГИБНЫМИ НАПРЯЖЕНИЯМИ ПРИ ПЕРЕСЕЧЕНИИ ИМИ ГЕОДИНАМИЧЕСКИХ ЗОН
}

\section{Аскаров Роберт Марагимович',}

askarov1943@mail.ru

\author{
Китаев Сергей Владимирович', \\ svkitaev@mail.ru
}

\author{
Исламов Ильдар Магзумович², \\ mildarislamov@mail.ru
1 Уфимский государственный нефтяной технический университет, Россия, 450064, г. Уфа, ул. Космонавтов, 1.
2 Ургалинское линейное производственное управление магистральных газопроводов, \\ Россия, 452570, Белокатайский р-н, с. Ургала, ул. Ленина, 93.
}

\begin{abstract}
Актуальность. Внутри Земли происходят термоядерные реакции, вызывающие перемещение земной поверхности. В областях сдвига тектонических плит - геодинамических зонах - происходит изменение положения подземных магистральных трубопроводов. В свою очередь изменение положения магистрального трубопровода приводит к изменению его напряженно-деформированного состояния. После активации разломов начинается движение блоков. Вначале они движутся медленно, затем темп движения возрастает, при этом в зону риска попадают трубопроводы, находящиеся в условиях длительной эксплуатации, поэтому вопрос контроля напряженно-деформированного состояния трубопроводов на пересечениях с геодинамическими зонами является актуальным.

Цель: разработать и внедрить технологию выявления участков трубопроводов при их пересечении с геодинамическими зонами, предусматривающую определение потенциально опасных участков, оценку напряженно-деформированного состояния трубы. объекты: области прохождения магистральных трубопроводов по территории Республики Башкортостан на пересечениях с геодинамическими зонами.

Методы: анализ результатов внутритрубной дефектоскопии с измерением радиусов изгиба трубопроводов, выявление потенциально-опасных участков, аналитический расчет напряженно-деформированного состояния трубопроводов.

Результаты. Изложена технология выявления участков трубопроводов с изгибными напряжениями при их пересечении с геодинамическими зонами. Технология предусматривает определение фактического положения магистрального трубопровода, непосредственное измерение радиуса изгиба. Располагая данными по участкам трубопроводов с ненормативными радиусами изгиба 500D и менее из отчетов по внутритрубной дефектоскопии, предлагается выявлять потенциально опасные участки, а повторный пропуск внутритрубных дефектоскопов каждые 2-3 года позволит оценивать динамику изменения радиусов изгиба, а также выявлять активные геодинамические зоны.
\end{abstract}

\section{Ключевые слова:}

Магистральный трубопровод, геодинамическая зона, напряженно-деформированное состояние, потенциально-опасный участок, радиус изгиба.

\section{Введение}

Среди инженерных сооружений подземные трубопроводные системы наиболее тесно связаны с окружающей средой. Объективная реальность такова, что пролегание трубопроводов неизбежно связано с пересечением многочисленных геодинамических зон (ГДЗ) [1-4], к которым можно отнести: разломы разного характера, движения земных блоков, надвигов (горных ударов), карсты и т. п.

Геодинамический фактор в большей или меньшей степени имеет место на всей поверхности земного шара, в т. ч. и в Республике Башкортостан (РБ) [5].

В настоящее время установлено, что причиной самой масштабной аварии XX в. в трубопроводном транспорте в районе поселка Улу-Теляк РБ, унесшей сотни жизней, является геодинамический фактор [5].

Вследствие термоядерных реакций внутри планеты и кругового движения вокруг солнца проис- ходят сдвиги земной поверхности [6-9]. На территории РБ были выявлены надвиги на протяжении 400 км, с образованием зон деформаций (рис. 1) $[5]$.

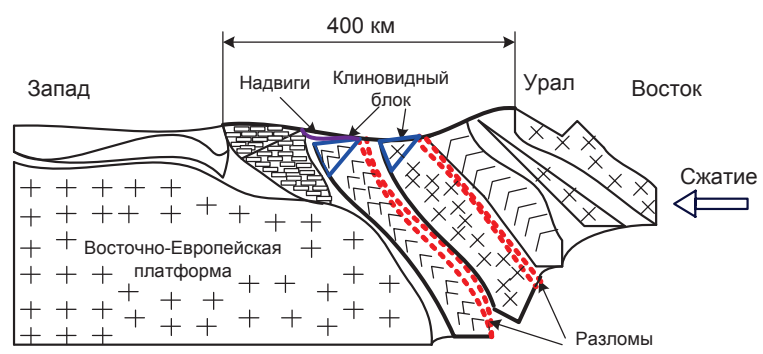

Pис.1. Схема движения надвигов по территории Республики Башкортостан (белой стрелкой указано направление действия сжимающей силы)

Fig. 1. Scheme of movement of thrusts on the territory of the Republic of Bashkortostan (white arrow indicates the direction of the compressive force action) 
При надвигах образуются складки местности, которые вовлекают в движение подземные трубопроводы (выделены синим цветом, условно на поверхности земли), воздействуя на их напряженнодеформированное состояние с возникновением зон деформаций (рис. 2).

В зависимости от направления движения тектонических плит могут быть рассмотрены следующие случаи движения соседних блоков [5]:

- вверх с разными скоростями в одном направлении;

- вниз с разными скоростями в одном направлении;

- с одинаковыми скоростями: оба опускаются либо поднимаются;

- в противоположных направлениях.

Это означает, что на пересечениях с ГДЗ неизбежно ее воздействие на трубопровод. В приведенных в работе [10] теоретических исследованиях вертикальное движение блоков названо «активными тектоническими разломами» (АТР). Опасность АТР заключается в вероятных относительных смещениях грунтовой массы в области разлома, при этом происходит передача нагрузок, превышающих проектные, на трубопровод.

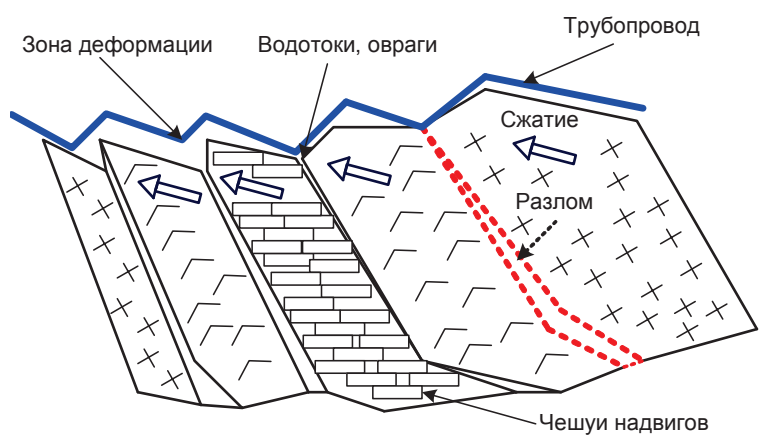

Pис. 2. Тектоническая схела надвигов с нанесенныли зонали де формаций на трубопроводах, в основном по переходам че рез водотоки (бельли стрелкали указано направление надвигов земных блоков)

Fig. 2. Tectonic scheme of overthrusts with deformed zones of deformation on pipelines, mainly on transitions through water courses (white arrows indicate the direction of earth thrusts)

Для трубопроводов к основным «аварийным» участкам относятся разломы (границы блоков).

При переходе из пассивного состояния в активное происходит активация разломов. В начальный период времени движение блоков происходит медленно, и после достижения определенных сжимающих усилий движение блоков ускоряется. Поэтому в большей степени перемещениям подвержены МТ со значительными сроками эксплуатации, например 33 года и более. При этом особую важность приобретает оценка напряженно-деформированного состояния (НДС) и прогноз технического состояния МТ, находящихся на пересечениях с ГДЗ. Нормативные документы по строительству и эксплуатации подземных МТ, которые определяют его НДС, основываются на теории неподвижной земли $[11,12]$, т. е. не учитывают геодинамический фактор.

Таким образом, возникла необходимость создания технологии, позволяющей раскрыть объективную картину линейно-высотного положения линейной части MT, выявлять потенциально опасные участки, в т. ч. на пересечениях с активными гдЗ.

В настоящее время в трубопроводном транспорте основной концепцией поддержания надежности является эксплуатация МТ по техническому состоянию (ТС), при этом ТС определяется по данным диагностики. Основным средством диагностики МТ в настоящее время является внутритрубная дефектоскопия (ВТД). Если наделить ВТД возможностью выявления потенциально-опасных участков (ПОУ) на всем протяжении линейной части MT, можно будет идентифицировать пересечения MT с ГДЗ. Технология ВТД постоянно совершенствуется, например, за последние 5-8 лет была оснащена навигационной системой, которая непрерывно фиксирует линейно-высотные отметки пути, что позволяет строить план и профиль обследованного участка $[13,14]$. Таким образом, появляется возможность отразить рельеф местности, так как вероятность возникновения ПОУ на ровном участке МТ минимальна.

Согласно [15], все ГДЗ, выявленные с помощью космических и обследованные наземными геологогеофизическими технологиями, представляют собой: реки с долинами, овраги, балки и т. п. (отрицательный рельеф местности), которые и составляют узлы границ разломов. В качестве примера, на рис. 3 приводится профиль участка, построенный по данным ВТД, с двумя ГДЗ.

Потенциальную опасность для участка МT представляют собой активные перемещающиеся ГДЗ. Даже внешне профиль этих ГДЗ (рис. 3) напоминает схематический профиль трубопровода на рис. 2 - клиновидные надвиги протяженностью около 5,0 км и 2,0 км.

В качестве расчетной схемы для оценки НДС MT на пересечениях с ГДЗ приняты две тектонические плиты, смещающиеся в вертикальной плоскости одна относительно другой на значение $\delta$ (полубесконечные пространства). Смещающиеся плиты вовлекают в движение трубопровод, проложенный в этой зоне. Принято допущение, что происходит одновременное смещение на одну и ту же величину на значительном удалении от оси разлома концов анализируемого участка трубопровода. На рис. 4 проиллюстрирована схема перемещения трубопровода в вертикальной плоскости при тектоническом разломе.

Перемещающийся вместе с массой грунта трубопровод одновременно сопротивляется его сдвигу. Это приводит к появлению нагрузок в зоне их контакта за счет смещения трубопровода и грунта относительно друг друга. При этом радиус кривизны оси трубопровода увеличивается или уменьшается за счет возникновения нагрузок при изгибе в 


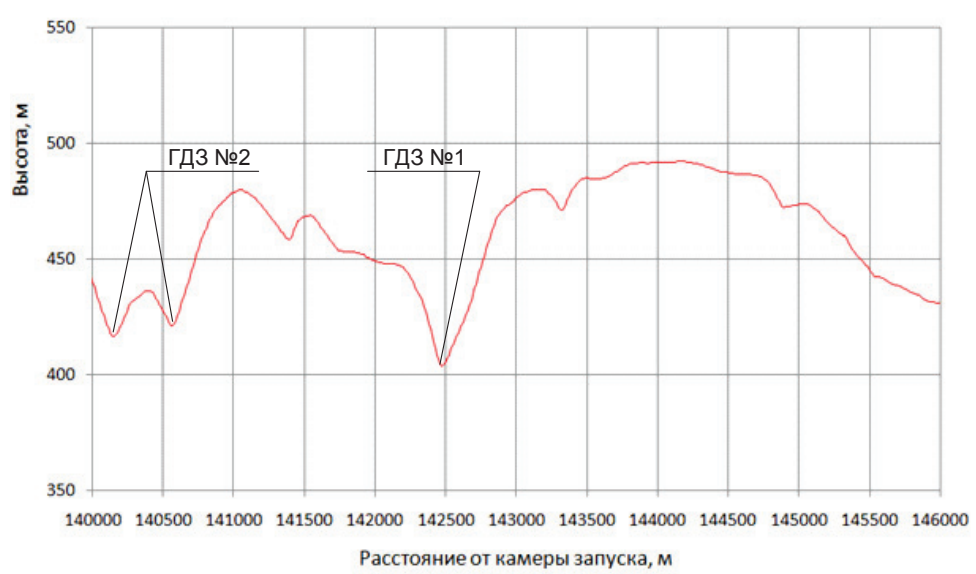

Рис. 3. Профиль участка магистрального трубопровода диаметром 1420 мм с двумя геодинамическими зонами № 1, 2

Fig. 3. Profile of the main pipeline section with a diameter $1420 \mathrm{~mm}$ with two geodynamic zones no.1,2

зоне движения [16]. На удалении от оси разлома происходит увеличение радиуса кривизны трубопровода, стремящейся к прямой линии, а изгибные напряжения приближаются к нулю.

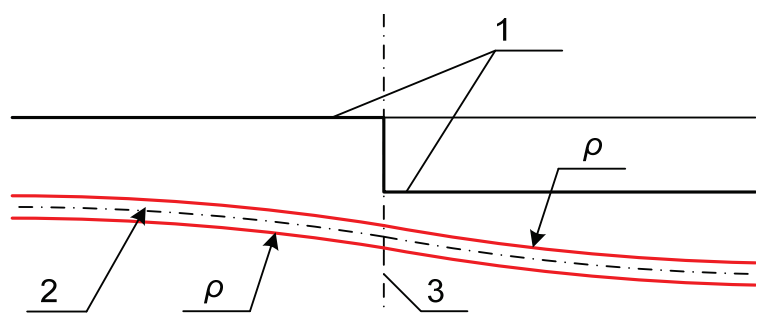

Pис. 4. Схема перемещения трубопровода в вертикальной плоскости при тектоническом разломе: 1 - поверхность земли; 2 - ось трубопровода; 3 - ось разлома

Fig.4. Scheme of pipeline movement in the vertical plane under a tectonic fault: 1 is the earth surface; 2 is the pipeline axis; 3 is the fault axis

На переходных участках вследствие смещения осей образуется радиус упругого изгиба $\rho$ (рис. 4 ). Примерно по оси разлома изгибные напряжения меняют знак, с выпуклого вверх на выпуклый вниз [17].

Таким образом, если появится возможность измерить радиус изгиба и его координаты, то можно с высокой степенью вероятности выявить ПОУ МТ и провести оценку его технического состояния на пересечениях с ГДЗ.

\section{Методика выявления потенциально \\ опасных участков магистральных трубопроводов и оценка их технического состояния}

Максимальные суммарные продольные напряжения от нормативных нагрузок и воздействий, согласно [11], для упруго-изогнутых и прямолинейных участков для подземно проложенных трубопроводов, рассчитываются по формуле:

$$
\sigma_{\text {пр }}^{\mathrm{H}}=\mu \sigma_{\text {кц }}^{\mathrm{H}}-\alpha \AA \Delta t \pm \sigma_{\text {й }},
$$

где $\Delta t$ - температурный перепад (расчетный) (принимается при нагревании положительным), ${ }^{\circ} \mathrm{C} ; \mu$ коэффициент Пуассона (поперечной деформации); $\sigma_{\text {кц }}^{\sharp}$ - кольцевые напряжения, возникающие от давления газа внутри трубы, МПа; $\alpha$ - коэффициент линейного расширения; $\sigma_{\text {u }}$ - напряжение упругого изгиба, МПа, рассчитывается по формуле:

$$
\sigma_{\mathrm{H}}=\mathrm{ED} / 2 \rho,
$$

$\rho$ - радиус упругого изгиба участка трубопровода; $E$ - модуль упругости для стали трубы, МПа; $D$ внутренний диаметр трубопровода, мм.

Анализ формулы (1) показывает, что первые две составляющие влияют главным образом на продольную силу, и только через нее - на продольные напряжения. Любое отклонение от прямолинейности приводит к изменению $\rho$, а значит отражается на величине продольных напряжений. Таким образом, непосредственное измерение радиуса упругого или упруго-пластического изгиба $\rho$, например, в процессе проведения ВТД, позволяет выявить ПОУ и получить первичную оценку их НДС по формуле (2). Такой метод, реализованный путем анализа деформации конструкции (в нашем случае трубопровода), можно считать прямым способом измерения НДС [18].

Данный метод обладает простотой, надежностью, достаточной точностью (от 4000 м) и позволяет контролировать весь трубопровод, а не только отдельные его части, доступные к наружному обследованию. Метод позволяет проводить диагностику НДС МТ без остановки транспортировки продукта и вскрытия трубопровода [18].

При проектировании линейной части магистральных трубопроводов участки прямолинейные и проложенные упругим изгибом, в соответствии с [15], не должны быть радиусом менее 1000D, т. е. если выявляется радиус изгиба менее $1000 \mathrm{D}$, можно отнести его к непроектному.

На рис. 5 в качестве примера приводится график измеренных радиусов упругого изгиба прямолинейного участка МГ диаметром 1420 мм, с выделением радиусов упругого изгиба $1000 \mathrm{D}$, полученный при плановой внутритрубной дефектоскопии. По оси абсцисс указано расстояние от камеры запуска, по оси ординат показан радиус кривизны трубопровода в диаметрах. 


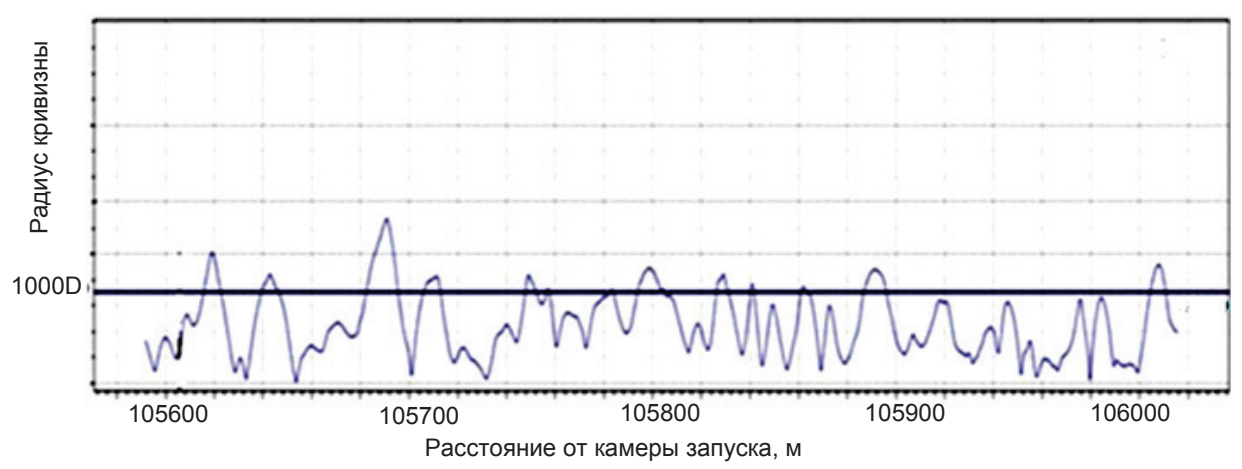

Рис. 5. График радиусов изгиба прямолинейного участка магистрального трубопровода

Fig. 5. Graph of bending radii of a straight section of the main pipeline

Из рис. 5 видно, что на прямолинейном участке MT протяженностью 400 м со сроком эксплуатации около 35 лет имеется 11 участков с радиусом упругого изгиба менее $1000 \mathrm{D}$, т. е. налицо фактическое несоответствие требованиям [15].

Оценивая требование 1000D [15], его, безусловно, можно считать обоснованным применительно к новому строительству, но по умолчанию оно переносится на весь срок эксплуатации МТ, главным образом потому, что механизма контроля этого параметра до последнего времени не существовало. В связи с появлением новой технологии выявления фактических радиусов упругого и упруго-пластического изгиба МТ это требование вступает в противоречие с его фактическим состоянием.

В [19] приводится научное обоснование критериев оценки опасности радиусов изгиба $\rho$ :

- 500D и выше - область, где трубная сталь гарантированно сохраняет упругие свойства;

- 250D и менее, что при переводе в изгибные напряжения составляет около 400 МПа и более, в соответствии с [15] являются недопустимыми;

- (250-500) D - область, требующая аналитической оценки в каждом конкретном случае.
C 2015 г. эти положения отражены в отчетах ВТД НПЦ Внутритрубная диагностика (основная подрядная организация ПАО «Газпром» по внутритрубной диагностике), где на обследованном участке указываются все выявленные участки с радиусом изгиба 500D и менее. Информация содержит: линейную координату, порядковый номер трубы, минимальный радиус (м) (включая отводы холодного гнутья (ОХГ)), минимальный радиус изгиба в диаметрах, его направление в часовых координатах. В качестве примера на рис. 6 приводится график радиусов изгиба на пересечении с ГДЗ, по данным ВТД 2015 г. ГДЗ представляет собой овраг с координатами 78810 м [20]. В области пересечения оврага участок МТ выполнен из двух отводов холодного гнутья (ОХГ).

Из рис. 6 видно, что на участке протяженностью 470 м ( $78580 \ldots 79050)$ имеются 3 участка с радиусами изгиба менее 500D с координатами $78706,8,4 ; 78775,4$ и 78919,8 м.

Плановые обследования средствами ВТД каждые 2-3 года позволяют оценить динамику изменения радиусов изгиба. В таблице приводятся сравнительные данные ВТД за 2015 и 2017 гг. Па-

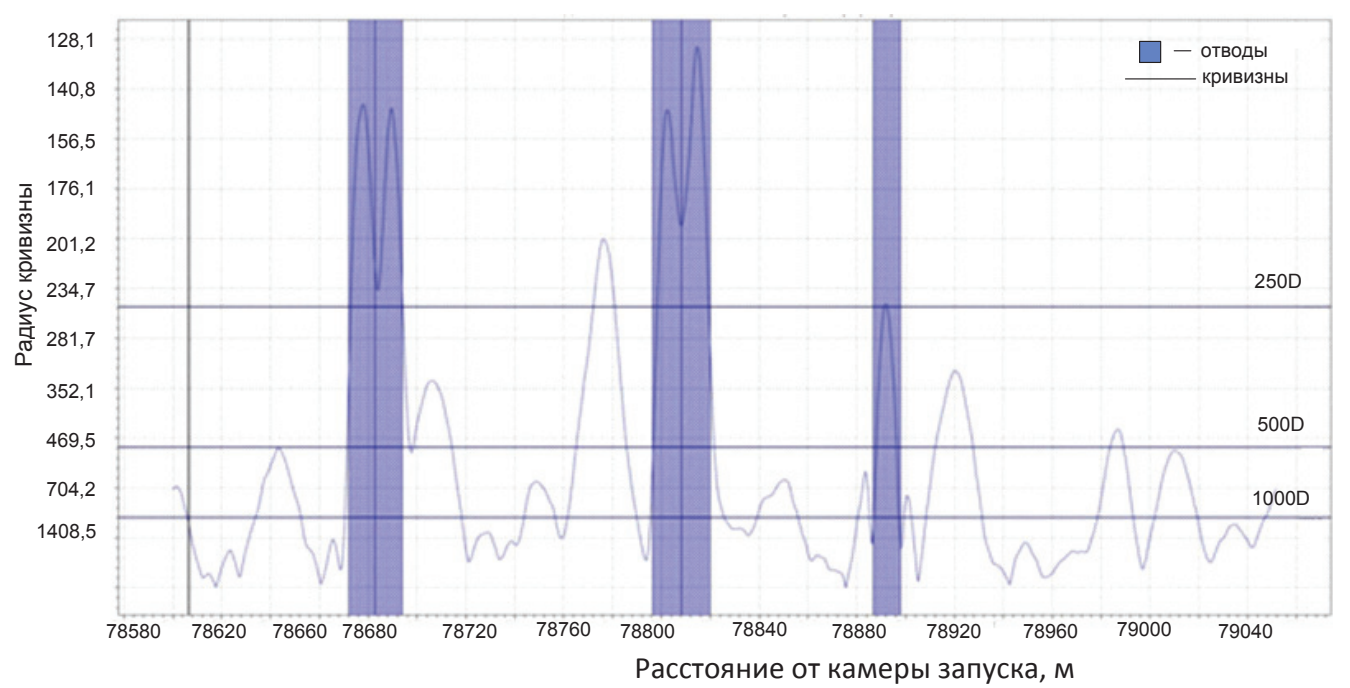

Pис. 6. График радиусов изгиба участка магистрального трубопровода на пересечении с геодинамическими зонами

Fig. 6. Graph of bend radii of the trunk pipeline section at the intersection with geodynamic zones 
раметры транспорта газа при пропуске снарядов ВТД: давление 6,6 МПа, температура газа $15,5^{\circ}$ и $12^{\circ}$.

таблица. Сравнительные данные по результатам внутри трубной дефектоскопии за 2015 и 2017 г2.

Table. $\quad$ Comparative data on in-pipe flaw detection results for 2015 and 2017

\begin{tabular}{|c|c|c|c|c|c|c|}
\hline $\begin{array}{c}\text { Номер } \\
\text { трубы } \\
\text { Pipe number }\end{array}$ & $\begin{array}{c}\text { Радиусы изгиба, в } \\
\text { диаметрах } \\
\text { Radii of bending, } \\
\text { in diameters }\end{array}$ & \multicolumn{2}{|c|}{$\begin{array}{c}\text { Линейная } \\
\text { координата, } \\
\text { Linear coordinate, } \\
\text { m }\end{array}$} & $\begin{array}{c}\text { Часовая коорди- } \\
\text { ната, часов } \\
\text { Hourly coordina- } \\
\text { tes, hours }\end{array}$ \\
\hline & 2015 & 2017 & 2015 & 2017 & 2015 & 2017 \\
\hline 7125 & $286 \mathrm{D}$ & $331 \mathrm{D}$ & 78706,8 & 78706,2 & 6,0 & 6,2 \\
\hline 7133 & 238D & 216D & 78775,4 & 78775,0 & 6,2 & 6,5 \\
\hline 7144 & 369D & 382D & 78919,8 & 78921,5 & 0,2 & 11,9 \\
\hline
\end{tabular}

На рис. 7 приводится укрупненный график радиусов изгиба МГ на пересечениях с ГДЗ № 16a, где отражены радиусы изгибов менее 500D (укрупненный в данном случае означает без учета радиусов на участках, где они менее 500D).

В верхней части эпюры указаны участки с выпуклостью вверх с 9 до 3 часов, в нижней - выпуклостью вниз с 3 до 9 часов.

Из данных двух пропусков ВТД (2015 и 2017 гг.), приведенных в таблице и на рис. 7, следует, что за два года [21]:

- линейные и часовые координаты непроектных радиусов изгиба практически не изменились (в пределах погрешности), что свидетельствует о совместимости результатов;

- понижение температуры между пропусками снаряда ВТД на $3,5^{\circ}$, в соответствии с формулой (1), могло вызвать увеличение растягивающей силы, а значит увеличить радиус непроектного изгиба, т. е. снизить изгибные напряжения;

- заметно изменение именно радиуса изгиба, на трубе № 7133 (в центре оврага) радиус изгиба уменьшился с 238D до 216D (10 \%), на двух других участках радиус изгиба увеличился: № 7125 с 286D до 331D (16 \% ), № 7144 с 369D до $382 \mathrm{D}(3 \%)$.

Изгибные напряжения, рассчитанные по формуле (2), в районе трубы № 7133, и без этого ненормативные, выросли с 444 до $486 \mathrm{MПа,} \mathrm{это} \mathrm{означа-}$ ет - участок подлежит ремонту.

Можно утверждать, что рассмотренное ГДЗ активное, именно активность ГДЗ, выраженная или в опускании средней части (дно оврага), или в подъеме с двух сторон бортов оврага отразилась на радиусах изгиба. В свою очередь появляется возможность выявления собственно активных ГДЗ, пересекающих МТ.

Изменения радиусов изгиба показывают, что трубопровод перемещается за окружающим его грунтом и это отражается в точках изгиба (перегиба), которые и представлены в виде радиусов изгиба. К ПОУ могут быть отнесены участки с тенденцией уменьшения зафиксированных радиусов изгиба, когда они находятся на границе, например $250 \mathrm{D}$.

\section{Заключение}

1. Вследствие термоядерных реакций внутри планеты и вращательного движения планеты вокруг солнца происходят движения земной поверхности (геодинамические зоны), которые вовлекают в него подземные магистральные трубопроводы. В свою очередь изменение положения МТ приводит к изменению его напряженно-деформированного состояния.

2. Таким образом, возникла необходимость создания технологии, позволяющей выявлять фактическое положение МТ, а значит и потенциально опасные участки на пересечениях с гдЗ.

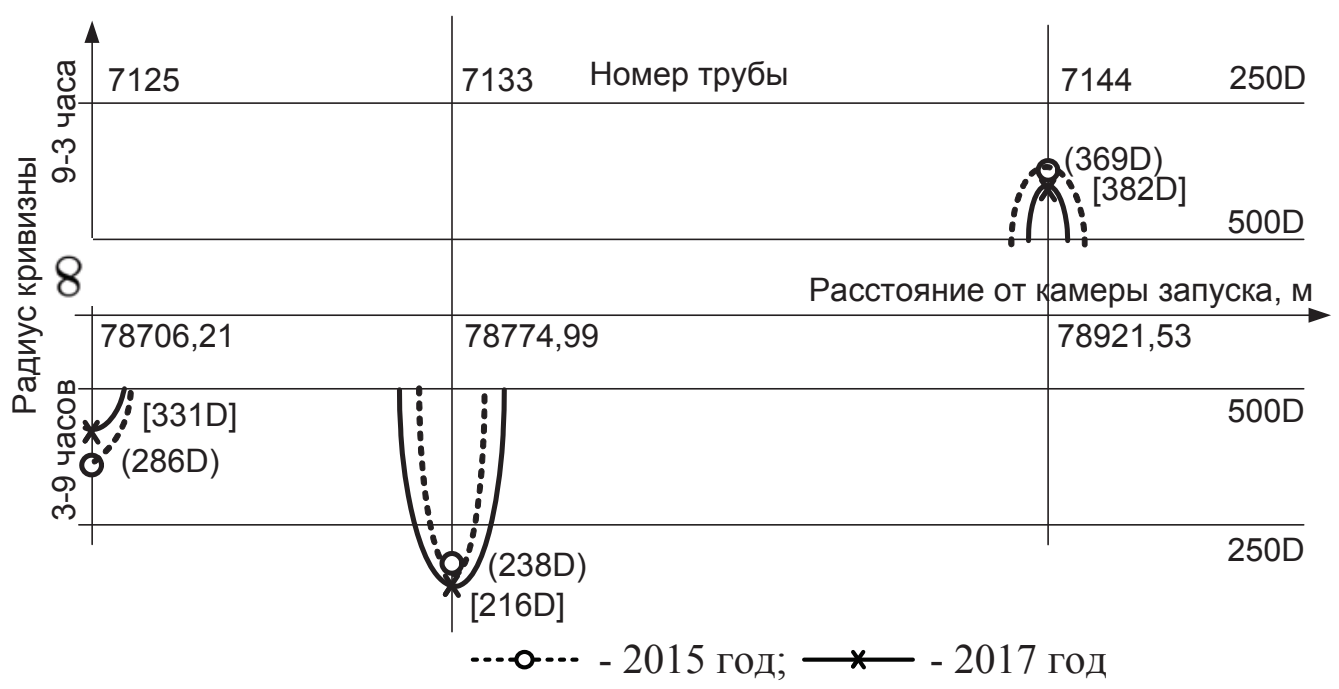

Pис. 7. Эпюра радиусов изгиба геодиналических зон

Fig. 7. Diagram of bending radii of geodynamic zones 
3. Разработка и внедрение технологии измерения радиусов изгиба МТ плановыми средствами ВТД, заключающейся в непосредственном измерении радиуса изгиба, позволяет выявить потенциально опасные участки и получить первичную оценку их НДС.

4. Обследования МТ по этой технологии показало, что требования нормативов по минимально допустимому радиусу изгиба $1000 \mathrm{D}$ и более после

\section{СПИСОК ЛИТЕРАТУРЫ}

1. Late Cenozoic magmatic inflation, crustal thickening, and $>2 \mathrm{~km}$ of surface uplift in central Tibet / J.L. Chen, A. Yin, J.F. Xu, Y.H. Dong, Z.Q. Kang // Geology. - 2018. - V. 46. - P. 19-22.

2. Haproff P.J., Zuza A.V., Yin A. West-directed thrusting south of the eastern Himalayan syntaxis indicates clockwise crustal flow at the indenter corner during the India-Asia collision // Tectonophysics. - 2018. - V. 722. - P. 277-285.

3. 3D geodynamic models for the development of opposing continental subduction zones: The Hindu Kush-Pamir example / J. Liao, T. Gerya, M. Thielmann, A.A.G. Webb, S.-K. Kufner, A. Yin // Earth and Planetary Science Letters. - 2017. - V. 480. P. 133-146.

4. Late Paleozoic and Mesozoic evolution of the Lhasa Terrane in the Xainza area of southern Tibet / S.Y. Fan, L. Ding, M.A. Murphy, M. Yao, A. Yin // Tectonophysics. - 2017. - V. 721. P. $415-434$.

5. Давлетов М.И. Исходные параметры для расшифровки геологических факторов аварий трубопроводов на территории Башкортостана // Энергоэффективность. Проблемы и решения: V Российский энергетический форум. - Уфа, 2005. - С. 232-237.

6. Duan B., Liu L., Yin A. Seismic shaking in the North China Basin expected from ruptures of a possible seismic gap // Geophysical Research Letters. - 2017. - V. 44. - P. 4855-4862.

7. The Himalaya in 3D: Slab dynamics controlled mountain building and monsoon intensification / A.A.G. Webb, H.C. Guo, P.D. Clift, L. Husson, T. Müller, D. Costantino, A. Yin, Z.Q. Xu, H. Cao, Q. Wang // Lithosphere. - 2017. - V. 9. - P. L636-1.

8. Spacing and strength of active continental strike-slip faults / A.V. Zuza, A. Yin, J. Lin, M. Sun // Earth and Planetary Science Letters. - 2017. - V. 457. - P. 49-62.

9. Pre-Cenozoic geologic history of the central and northern Tibetan Plateau and the role of Wilson cycles in constructing the Tethyan orogenic system / C. Wu, A. Yin, J.Y. Zhang, W.C. Liu, L. Ding // Lithosphere. - 2016. - V. 8. - P. 254-292.

10. Фигаров Э.Н. Оценка напряженного состояния подземного трубопровода, пересекающего зоны активных тектонических разломов // Трубопроводный транспорт: теория и практика. 2012. - № 6. - C. 39-42.

11. СП 36.13330.2012 Магистральные трубопроводы (Актуализированная редакция СНиП 2.05.06-85* с изменениями и дополнениями). URL: http://docs.cntd.ru/document/1200103173 (дата обращения: 13.05.2018). эксплуатации за рамками амортизационного срока преимущественно не выполняются.

5. В отчетах ВТД указываются все участки ЛЧ МГ с ненормативными радиусами изгиба 500D и менее, что позволяет выявлять потенциально опасные участки. По результатам пропуска снарядов ВТД с периодичностью раз в 2-3 года производится оценка динамики изменения радиусов изгиба, при этом выявляются активные ГдЗ.

12. СП 86.13330.2012. Магистральные трубопроводы (Актуализированная редакция СНиП III-42-80*). URL: http://docs. cntd.ru/document/1200102566 (дата обращения: 13.05.2018).

13. Successful management of the pipeline cracking threat using an ultrasonic in-line inspection tool - a case study / B. Delanty, I. Whitbread, M. Maxwell, J. Munro // Pipelines international. 2015. - № 9. - P. 44-48.

14. Pipeline Operators Forum. Specifications and requirements for intelligent pig inspection of pipelines. Version 2016. - 2016. 56 p. URL: file://D:/User/\% D0 \% $97 \%$ D0 \% B0 \% D0 \% B3 \% D1 \% 80 \% D1 \% 83 \% D0 \% B7 \% D0 \% BA \% D0 \% B8/publicdocuments 01 2016 Version.pdf (дата обращения 20.05.2019).

15. Аскаров Р.М.,., Мазитов Д.Г., Рафиков С.К. Прогноз напряженнодеформированного состояния участков газопроводов, пересекающих геодинамические зоны // Проблемы сбора, подготовки и транспорта нефти и нефтепродуктов. - 2015. - № 1. - С. 121-126.

16. Айнбиндер А.Б. Расчет магистральных и промысловых трубопроводов на прочность и устойчивость. Справочное пособие. М.: Недра, 1991. - 287 с.

17. Писаренко Г.С., Яковлев А.П., Матвеев В.В. Справочник по сопротивлению материалов. - Киев: Изд-во «Наукова думка», 1988. $-736 \mathrm{c}$.

18. Способ определения потенциально опасного участка трубопровода с непроектным уровнем напряженно-деформированного состояния: заявка на изобретение № 2602327 РФ; заявл. 04.04.2015; опубл. 20.11.2016; Бюл. № 32. - 10 c.

19. 0 фактических радиусах изгиба линейной части магистральных газопроводов / Р.М. Аскаров, К.М. Гумеров, А.Н. Кукушкин, И.М. Исламов // Трубопроводный транспорт: теория и практика. - 2017. - № 6. - С. 28-33.

20. Выявление потенциально опасных участков магистральных газопроводов на пересечениях с геодинамическими зонами / Б.Н. Мастобаев, Р.М. Аскаров, С.В. Китаев, С.К. Рафиков, Р.Р. Усманов, М.В. Чучкалов, И.М. Исламов // Трубопроводный транспорт: теория и практика. - 2017. - № 3. - С. 38-43.

21. Напряженно-деформированное состояние газопровода на пересечениях с геодинамическими зонами по данным неоднократной внутритрубной дефектоскопии / Б.Н. Мастобаев, Р.М. Аскаров, С.В. Китаев, Р.М. Каримов, А.Р. Валеев, Т.А. Хакимов, И.М. Исламов // Транспорт и хранение нефтепродуктов и углеводородного сырья. - 2017. - № 6. - С. 50-57.

Поступила 27.05.2018 г.

\section{Информация об авторах}

Аскаров P.M., доктор технических наук, доцент кафедры транспорта и хранения нефти и газа Уфимского государственного нефтяного технического университета.

Китаев C.B., доктор технических наук, профессор кафедры транспорта и хранения нефти и газа Уфимского государственного нефтяного технического университета.

Исламов И.М., начальник Ургалинского линейного производственного управления магистральных газопроводов. 


\title{
ON TECHNOLOGY OF DETECTING PIPELINE SITES WITH BENDING VOLTAGES IN THEIR CROSSING GEODYNAMIC ZONES
}

\author{
Robert M. Askarov' \\ askarov1943@mail.ru \\ Sergey V. Kitaev', \\ svkitaev@mail.ru \\ Ildar M. Islamov², \\ mildarislamov@mail.ru
1 Ufa State Petroleum Technological University, 1, Kosmonavtov street, Ufa, 450064, Russia.
2 Urgala linear production office of main gas pipelines, \\ 93, Lenin street, Urgala, 452570, Russia.
}

\begin{abstract}
The relevance. There are thermonuclear reactions inside the Earth. This causes displacement of the earth's surface. In the areas of shear tectonic plates - geodynamic zones - there is a change in position of underground pipelines. Changing the position of the main pipeline leads in its turn to a change in its stress-strain state. After activation of the faults, the blocks start moving. At first they move slow$l y$, then the rate of movement increases, the pipelines that are under long-term operation get into the risk zone, so the control of the stress-strain state of the pipelines at the intersections with the geodynamic zones is relevant.

The main aim of the research is to develop and implement a technology for identifying sections of pipelines at their intersection with geodynamic zones, providing for identification of potentially hazardous areas to assess the stress-strain state of the pipe.

The objects: areas of passage of the main pipelines on the territory of the Republic of Bashkortostan at the intersections with geodynamic zones.

Methods: analysis of the results of in-line inspection with measurement of bending radii of pipelines, identification of potentially dangerous areas, analytical calculation of the stress-strain state of pipelines.

Results. The paper introduces the technology of revealing sections of pipelines with flexural stresses at their intersection with geodynamic zones. The technology involves determination of actual position of the main pipeline, direct measurement of the bend radius. Having the data on the sections of pipelines with non-normative bending radii of 500D and less the authors have proposed to identify potentially hazardous areas from the reports on in-line flaw detection in the work; re-skip of in-tube flaw detectors, every 2-3 years will allow estimating the dynamics of the change in bend radii, and identifying as well active geodynamic zone.
\end{abstract}

Key words:

Main pipeline, geodynamic zone, stress-strain state, potentially dangerous area, bending radius.

\section{REFERENCES}

1. Chen J.L., Yin A., Xu J.F., Dong Y.H., Kang Z.Q. Late Cenozoic magmatic inflation, crustal thickening, and $>2 \mathrm{~km}$ of surface uplift in central Tibet. Geology, 2018, vol. 46, pp. 19-22.

2. Haproff P.J., Zuza A.V., Yin A. West-directed thrusting south of the eastern Himalayan syntaxis indicates clockwise crustal flow at the indenter corner during the India-Asia collision. Tectonophysics, 2018, vol. 722, pp. 277-285.

3. Liao J., Gerya T., Thielmann M., Webb A.A.G., Kufner S.-K., Yin A. 3D geodynamic models for the development of opposing continental subduction zones: The Hindu Kush-Pamir example. Earth and Planetary Science Letters, 2017, vol. 480, pp. 133-146.

4. Fan S.Y., Ding L., Murphy M.A., Yao M., Yin A. Late Paleozoic and Mesozoic evolution of the Lhasa Terrane in the Xainza area of southern Tibet. Tectonophysics, 2017, vol. 721, pp. 415-434.

5. Davletov M.I. Iskhodnye parametry dlya rasshifrovki geologicheskikh faktorov avary truboprovodov na territorii Bashkortostana [Initial parameters for decoding geological factors of pipeline accidents in the territory of Bashkortostan]. Energoeffek tivnost. Problem i resheniya. V Rossiysky energetichesky forum [V Russian Energy Forum. Energy efficiency. Problems and solutions]. Ufa, 2005. pp. 232-237.

6. Duan B., Liu L., Yin A. Seismic shaking in the North China Basin expected from ruptures of a possible seismic gap. Geophysical Research Letters, 2017, vol. 44, pp. 4855-4862.
7. Webb A.A.G., Guo H.C., Clift P.D., Husson L., Müller T., Costantino D., Yin A., Xu Z.Q, Cao H., Wang Q. The Himalaya in 3D: Slab dynamics controlled mountain building and monsoon intensification. Lithosphere, 2017, vol. 9, pp. L636-1.

8. Zuza A.V., Yin A. Lin J., Sun M. Spacing and strength of active continental strike-slip faults. Earth and Planetary Science Letters, 2017, vol. 457, pp. 49-62.

9. Wu C., Yin A., Zhang J.Y., Liu W.C., Ding L. Pre-Cenozoic geologic history of the central and northern Tibetan Plateau and the role of Wilson cycles in constructing the Tethyan orogenic system. Lithosphere, 2016, vol. 8, pp. 254-292.

10. Figarov E.N. Assessment of the stressed state of an underground pipeline crossing zones of active tectonic faults. Pipeline transport: theory and practice, 2012, no. 6, pp. 39-42. In Rus.

11. SP 36.13330.2012 Magistralnye truboprovody (Aktualizirovannaya redaktsiya SNiP $2.05 .06-85$ * s izmeneniyami i dopolneniyami) [Trunk pipelines (updated version of SNiP 2.05.06-85* as amended)]. Available at: http://docs.cntd.ru/document/ 1200103173 (assessed: 13 May 2018).

12. SP 86.13330.2012 Magistralnye truboprovody (Aktualizirovannaya redaktsiya SNiP III-42-80*) [Trunk pipelines (Updated version of SNiP III-42-80*)]. Available at: http://docs.cntd.ru/document/1200102566 (assessed: 13 May 2018).

13. Delanty B., Whitbread I., Maxwell M., Munro J. Successful management of the pipeline cracking threat using an ultrasonic in-li- 
ne inspection tool - a case study. Pipelines international, 2015, no. 9 , pp. 44-48.

14. Pipeline Operators Forum. Specifications and requirements for in telligent pig inspection of pipelines. Version 2016. 2016. $56 \mathrm{p}$. Available at: file://D:/User/\% D0 \% $97 \%$ D0 \% B0 \% D0 \% B3 \% D1 \% $80 \%$ D1 \% $83 \%$ D0 \% B7 \% D0 \% BA $\%$ D0 \% B8/public-documents_01_2016_Version.pdf (accessed 20 May 2019).

15. Askarov R.M., Mazitov D.G., Rafikov S.K. Forecast of the stressstrain state of sections of gas pipelines crossing geodynamic zones. Problems of collecting, preparing and transporting oil and oil products, 2015, no. 1, pp. 121-126. In Rus.

16. Aynbinder A.B. Raschet magistralnykh i promyslovykh truboprovodov na prochnost $i$ ustoychivost. Spravochnoe posobie [Calculation of main and field pipelines for strength and stability. Reference Manual]. Moscow, Nedra Publ., 1991. 287 p.

17. Pisarenko G.S., Yakovlev A.P., Matveev V.V. Spravochnik po soprotivleniyu materialov [Handbook of material resistance]. Kiev, Naukova dumka Publ., 1988. 736 p.

18. Usmanov R.R., Chuchkalov M.V., Askarov R.M., Zakiryanov R.V. Sposob opredeleniya potentsialno opasnogo uchastka truboprovoda s neproektnym urounem napryazhenno-deformiro- vannogo sostoyaniya [A method for determining a potentially dangerous pipeline section with a non-design level of stress-strain state]. Patent RF no. 2602327, 2016.

19. Askarov R.M., Gumerov K.M., Kukushkin A.N., Islamov I.M. On the actual radii of the bending of the linear part of the main gas pipelines. Pipeline transport: theory and practice, 2017, no. 6, pp. 28-33. In Rus.

20. Mastobaev B.N., Askarov R.M., Kitaev S.V., Rafikov S.K., Usmanov R.R., Chuchkalov M.V., Islamov I.M. Identification of potentially hazardous sections of main gas pipelines at intersections with geodynamic zones. Pipeline transport: theory and practice, 2017, no. 3, pp. 38-43. In Rus.

21. Mastobaev B.N., Askarov R.M., Kitaev S.V., Karimov R.M., Valeev A.R., Xakimov T.A., Islamov I.M. The stress-strain state of the gas pipeline at the intersections with geodynamic zones from the data of repeated in-line inspection. Transportation and storage of petroleum products and hydrocarbons, 2017, no. 6 . pp. 50-57. In Rus.

Received: 27 May 2018.

\section{Information about the authors}

Robert M. Askarov, Dr. Sc., associate professor, Ufa State Petroleum Technological University.

Sergei V. Kitaev, Dr. Sc., professor, Ufa State Petroleum Technological University.

Ildar M. Islamov, the head of the Urgala linear production office of main gas pipelines. 